\title{
Modeling SEM Column, Probe Formation, and Imaging Using Fourier Optics
}

\author{
Surya Kamal and Richard Hailstone
}

Rochester Institute of Technology, United States

Resolution of a scanning electron microscope is theoretically limited by the electron probe dimensions at the specimen. Probe formation in SEM [1-2] has been well understood using both geometrical optics and wave optics of the electron beam. However, there is a need for a software simulation that equips the microscopist to visualize how the probe will look in different operating conditions of an SEM and with different degrees of aberrations. Given the specimen structure signal [3], this will enable the user to see how the probe affects the imaging. Our final aim is to suggest changes in the SEM itself so as to improve its imaging capabilities. For that, it is very important to be aware of the effects of probe size and shape on the imaging in detail.

In this work, we present new software that models a field emission SEM column and probe formation using Fourier optics; it produces an image of the specimen using the imaging model described by Sato [3]. It performs coherent and partially coherent imaging of the electron beam by the objective lens. Aberrations distort the probe and therefore both coherent (seidel\& parasitic) and incoherent (chromatic) aberrations were also simulated to realize their effect. It is important to note that we ensure that all input parameters to the simulation including the aberration coefficients are well known to a standard SEM user. For instance, $V_{o}$ is the accelerating voltage, $i_{p}$ is the probe current, $\beta$ is the gun brightness, and so on. Once these parameters are provided by the user, they are translated to Fourier optics equivalents; for instance, $V_{o}$ is used to find the wavelength $(\lambda)$ of the electrons.

The simulation experiments all agree with the expected shape and size of the probe under different conditions. In figure 1(a), we show how the point spread function (PSF) of the lens system and the probe intensity vary with accelerating voltage. For a $20 \mathrm{KV}$ voltage, we get the smallest probe which means much better resolution compared to other voltages. Figure 1(b) shows a typical probe intensity formed after the beam passes through the objective lens with aberrations. Now, when this aberrated probe interacts with the specimen, it produces a distorted final image which is shown in Figure 1(c).

In addition to these main features, the software also has the capability to add Poisson and Gaussian noise to the generated probe and the final specimen image. Once the final image is generated, we can also get back the probe intensity from which it was generated. This can be done using a simple Wiener-filter deconvolution [4] which is also included in the software. Possible applications for this software can be modeling aberration correction in probe formation, identification of aberrations present in an SEM by image analysis of the final specimen image, and understanding the effects of a different combination of operating conditions on imaging and resolution of the SEM. 


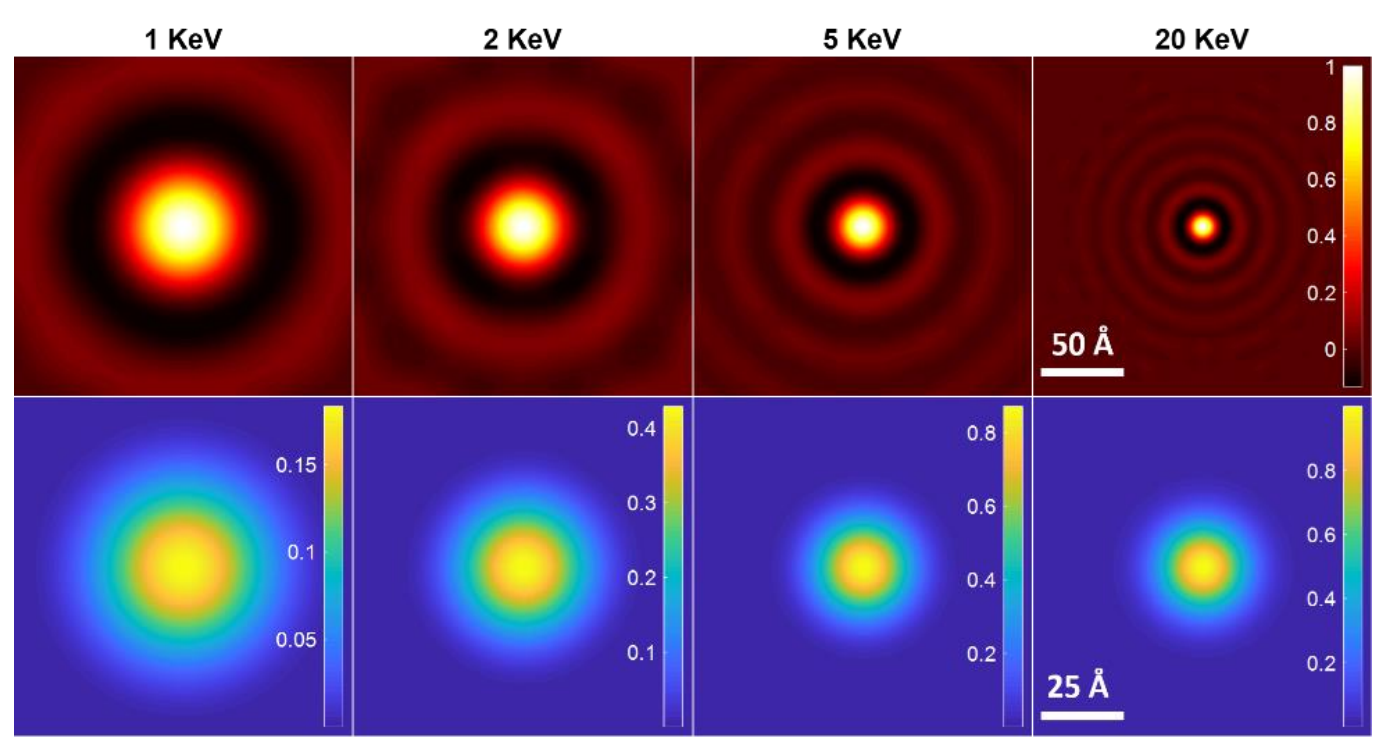

Figure 1. (a) PSF of the electron lens system and probe intensity at the specimen variation w.r.t beam voltage. (b) A typical aberrated probe intensity at the specimen with $1 \lambda$-defocus, $2 \lambda$ - pherical aberration, $7 \lambda$ - non-axial coma, top view (left), contour (center), 3D surface plot (right)

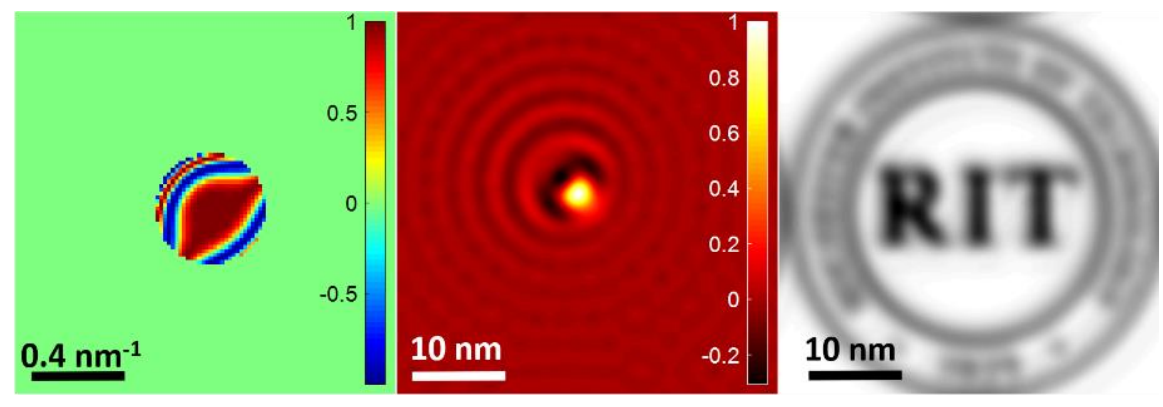

Figure 2. Coherent transfer function of the electron lens system distorted with aberrations (left), PSF of the electron lens system (center) and the final image generated for a RIT object using the aberrated probe intensity (right) formed using the lens system.

\section{References}

[1] J. Ximen, Z. Shao, P.S.D. Lin, Journal of Microscopy, Vol. 170. Pt 2, May 1993, p. 119-124. [2] Reimer L. (1998) Electron Optics of a Scanning Electron Microscope. In: Scanning Electron Microscopy. Springer Series in Optical Sciences, vol 45. [3] Orloff, J. (Ed.). (2009) Chapter 8. In: Handbook of Charged Particle Optics (2nd ed.)

[4] Zotta, M., Nevins, M. et al. Microscopy and Microanalysis, 24(4) (2018), p. 396-405 